\title{
The Conundrum of Adjuvant HER2 Treatment Options
}

\author{
Ajaz Bulbul',2*, Emilio Araujo-Mino ${ }^{2,3}$ and Zoneddy Ruiz Dayao ${ }^{3}$ \\ ${ }^{1}$ Division of Internal Medicine, Department of Hematology/Oncology, Texas Tech University Health Sciences Center School of \\ Medicine, Lubbock, TX, United States, ${ }^{2}$ Hematology and Oncology, Kymera Cancer Center, Carlsbad, NM, United States, \\ ${ }^{3}$ Division of Hematology Oncology, University of New Mexico Comprehensive Cancer Center, Albuquerque, NM, United States
}

Keywords: HER2, neratinib, APHINITY, trastuzumab, pertuzumab, ExteNet

\section{INTRODUCTION}

Improving outcomes in HER2 over-expressing breast cancer has been an impressive success story over the years. Remarkable clinical benefit and large hazard ratios in earlier metastatic and adjuvant trials rendered hope to more contemporary adjuvant trials of a similar large-scale benefit. Recent FDA approvals of dual HER2-blockade with trastuzumab plus pertuzumab and neratinib based on the APHINITY and ExteNET trials, respectively, were modest and rather underwhelming. Future trials need to focus on identifying robust biomarkers and clinical parameters that can best define the subset of patients where the anticipated toxicities and cost of therapy are justified.

\section{OPEN ACCESS}

Edited by:

José Bines,

Instituto Nacional de Câncer

(INCA), Brazil

Reviewed by:

Alessandro Igor Cavalcanti Leal,

Johns Hopkins Medicine,

United States

Shigehira Saji,

Kyoto University, Japan

${ }^{*}$ Correspondence:

Ajaz Bulbu

ajazbulbu@gmail.com

Specialty section:

This article was submitted

to Women's Cancer,

a section of the journal

Frontiers in Oncology

Received: 06 February 2018

Accepted: 08 May 2018

Published: 30 May 2018

Citation:

Bulbul A, Araujo-Mino E and Dayao ZR (2018) The Conundrum of Adjuvant HER2 Treatment Options.

Front. Oncol. 8:177. doi: 10.3389/fonc.2018.00177

\section{ROLE OF DUAL HER2 BLOCKADE IN THE ADJUVANT TREATMENT}

APHINITY was an adjuvant study of 4,805 HER2-positive post mastectomy or lumpectomy patients randomly assigned to receive standard 18-week chemotherapy plus 1 year of either trastuzumab and placebo or trastuzumab and pertuzumab for tumors $>1 \mathrm{~cm}$ (Figure 1).

von Minckwitz et al. (1) and the APHINITY team showed a positive but disappointing $0.9 \%$ absolute benefit in invasive disease-free survival (iDFS) (HR 0.81). Even among higher risk nodepositive women, pertuzumab improved the 3-year disease-free survival (DFS) by only $1.8 \%$, from 90.2 to $92.0 \%$ (HR $0.77,95 \% ; P=0.02$ ). The modest benefit was mostly confined to the two-thirds of patients who were node positive and roughly the third who had HR-negative disease with an absolute benefit of 1.8 and 1.6\%, respectively, with the addition of pertuzumab at 3 years.

The recurrence rate was low in node-negative patients. Not surprisingly, there was no difference between the treatment arms. Similarly, in the HR-positive group, a benefit was not evident. Nodenegative enrollment accounted for $36 \%$ of patients which is higher than the $12 \%$ in the N9831 and B-31 (2), possibly diluting a potential benefit and accounting for the better than expected $93.2 \%$ 3-year DFS in the placebo group as compared to $86.7 \%$ in the N9831 and B-31 joint analysis (2) at 3.5 years, $88 \%$ with BCIRG006 at 3 years (3), and $85.8 \%$ at 2 years in HERA (4) and $91.6 \%$ a more contemporary ExteNET (5). In addition, APHINITY may have included lower risk patients in the beginning since later the protocol was amended to limit enrollment of high-risk node-negative and allow more node-positive disease patients when 3,655 out of 4,804 were already enrolled (Table 1).

We need to recognize that patients in this trial overall had an excellent prognosis as is expected now with contemporary adjuvant trials with standard chemotherapy/trastuzumab treatment. Large benefits are difficult to achieve, and many need to be treated to benefit a few. As an example, the APT trial in node-negative patients had an excellent 3-year DFS of $98.7 \%$ with only weekly paclitaxel with a year of trastuzumab dual blockade. It would be difficult to improve on these numbers in this cohort of low-risk patients (6). 


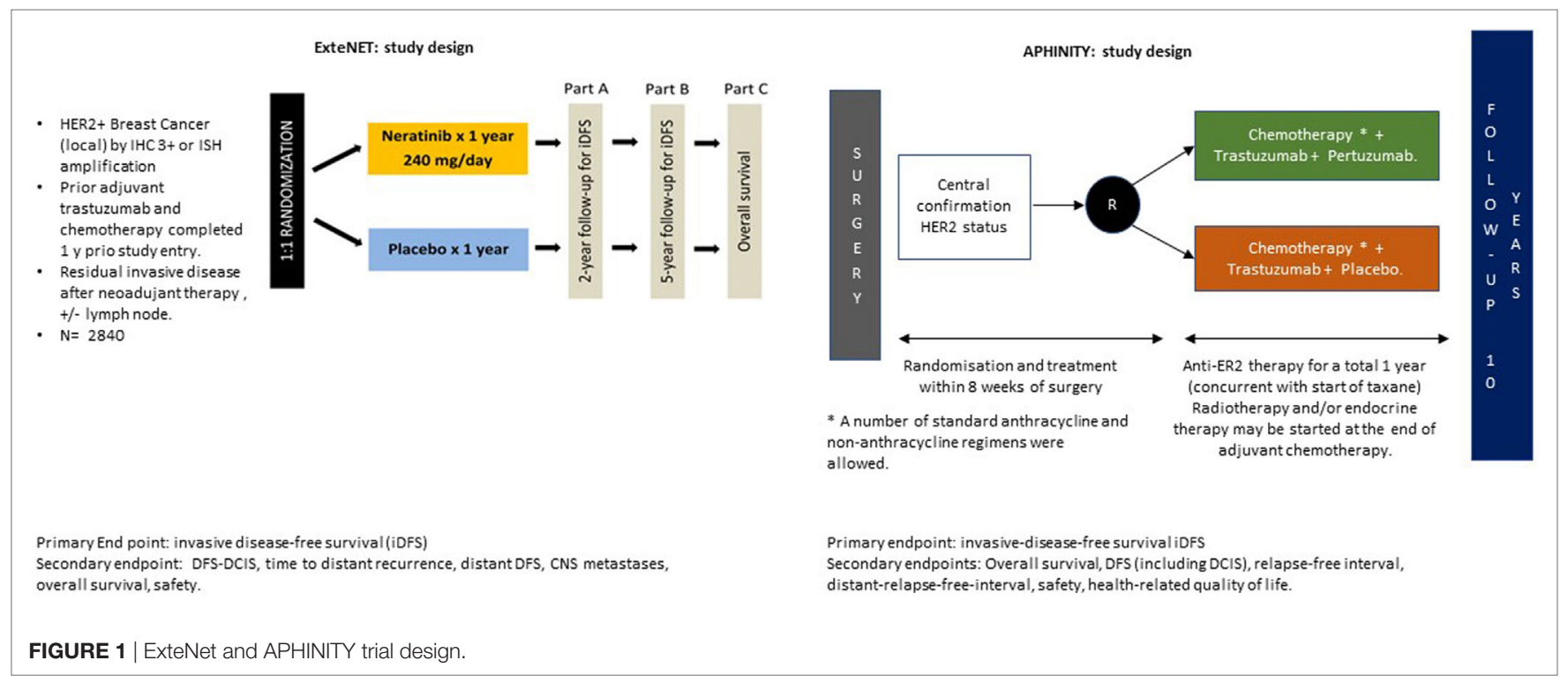

TABLE 1 | Selected adjuvant HER2 trials.

\begin{tabular}{|c|c|c|c|c|c|}
\hline Patient characteristics & BCIRG 006 & HERA & $\begin{array}{c}\text { Joint analysis NSABP B-31 } \\
\text { and NCCTG N9831 }\end{array}$ & APHINITY & ExteNet \\
\hline $\mathrm{ER}+(\%)$ & 54 & 50 & 53 & 64 & 57 \\
\hline ER- (\%) & 46 & 50 & 47 & 36 & 43 \\
\hline T1 (\%) & 40 & 39 & 41 & 40 & 31 \\
\hline T2 (\%) & 54 & 44 & 43 & 53 & 40 \\
\hline T3 (\%) & 6 & 5 & $14.4^{\mathrm{a}}$ & 6 & 9 \\
\hline NO (\%) & 28 & 32 & 13 & 38 & 24 \\
\hline N1 (\%) & 38 & 29 & 48 & 37 & 47 \\
\hline N2 (\%) & 23 & 28 & 24 & 25 & 30 \\
\hline N3 (\%) & 10 & NR & 13 & NR & NR \\
\hline \multicolumn{6}{|l|}{ Chemo regimen } \\
\hline Control arm & AC- $\mathrm{T}$ & Several chemo neo/adj & AC-T & Chemo + T & Several chemo + T neo/adj \\
\hline Experimental arm & $\begin{array}{l}\text { AC- } \mathrm{T}+1 \text { year } \\
\text { trastuzumab }(\mathrm{Tz})^{\mathrm{b}}\end{array}$ & Chemo + 1 year Tz & $A C-T+1$ year $T z$ & Chemo $+\mathrm{Tz}+\mathrm{P}$ & Chemo + Tz + neratinib \\
\hline Disease-free survival & 3 years: 81 vs 88 & 2 years: 87.4 vs 85.8 & 3 years: 75.4 vs 87.1 & 3 years: 93.2 vs 94.1 & 2 years: 91.6 vs 93.9 \\
\hline (DFS) (\%) & 5 years: 75 vs 84 & 4 years: 73 vs 78.3 & 4 years: 67.1 vs 85.3 & & \\
\hline Hazard ratio for DFS & 5 years: 0.64 & 4 years: 0.76 & 3 years: 0.48 & 3 years: 0.81 & 2 years: 0.67 \\
\hline Overall survival (\%) & 5 years: 87 vs 92 & 4 years: 87.4 vs 89.3 & 3 years: 91.7 vs 94.3 & NR & NR \\
\hline Hazar ratio for OS & 5 years: 0.63 & 4 years: 0.85 & 3 years: 0.67 & NR & NR \\
\hline
\end{tabular}

Staging, nodal status, and respective DFS.

a Tumors $>4 \mathrm{~cm}$.

${ }^{\circ} \mathrm{TCH}$ evaluated but not included in this table.

'2-Year trastuzumab was also evaluated.

$N R$, not reported.

Eight-year data from HERA trial showed the HR for DFS of 0.76 which was similar at 4 years and attenuated compared to the HR 0.54 at year 1 suggesting that there is a possibly diminishing benefit with a flattening of the DFS curve after an initial drop (4). These results are consistent with the magnitude of benefit seen when lapatinib was added to trastuzumab in the ALLTO trial ( 86 vs $88 \% 4$ years DFS) (7). In our opinion, therefore, it is unlikely that longer follow-up would magnify the benefit currently reported; except for possibly the high-risk node-positive patients; which would merit future follow-up of the trial.
The challenge lies in identifying who can benefit from therapy and avoid overtreatment. Future studies need to focus on evaluating molecular markers that elucidate the heterogeneous response to anti-HER2 agents and predict responses. Unfortunately, the biomarker analysis in TRYPHAENA (8), CLEOPATRA (9), and NeoSphere (10) were mostly negative with PIK3CA being mainly prognostic but not predictive and with limited power to detect correlations. High HER2 protein, HER2, and HER3 mRNA levels, wild-type PIK3CA, and low serum HER2 showed a significantly better prognosis $(P<0.05)$ (9). However, other trials show promising molecular signals. In 
the docetaxel, trastuzumab, pertuzumab arm of the NeoSphere trial, higher expression of immunogenic markers PD1 and STAT1, CTLA4, MHC1 were linked with lower pathological $\mathrm{CR}$ rate suggesting that the combination of anti-HER2 and immune-checkpoint inhibitors (11) is a reasonable strategy to explore. The magnitude of CD8+ tumor-infiltrating lymphocytes in TNBC and HER2 overexpressing tumors may help identify breast cancers with higher predicted response rates (12). Novel prognostic markers are similarly being explored. Higher anti-HER2 CD4+ T-helper type 1 response is a promising immune correlate to pathologic response. HER2-directed Th1 immune interventions like HER2 dendritic cell vaccine have shown mitigation of pCR rates in early studies (13). The PANACEA trial (NCT02129556) is currently evaluating this concept in the metastatic setting with trastuzumab and NCT03032107 with TDM-1. A CLEOPATRA'esque NCT03199885 design is evaluating pembrolizumab with paclitaxel and dual HER2 blockade.

Traditionally, identification of HER positivity is made by identification of overexpression or amplification of the HER2 gene (14). In the NSABP-47 trial, there was no benefit noted with trastuzumab in the low HER2 expressing tumors (IHC 1,2+, FISH $<2$ ). These validate that trastuzumab has no current role in the HER2-negative population as currently defined. However, somatic mutations in the HER2 gene, HER3, HER4 which may not be identified by traditional testing are sometimes functionally active mutations. It is unclear if these can be potentially targeted by current treatments $(15,16)$.

Adaptive immune mechanisms may have a role in modulating pCR with HER2-directed therapy in high PDL1 expressers (17). RNA sequencing data from NeoALLTO suggest that pCR was associated with high expression of ERBB2 and low expression of ESR1 (estrogen receptor 1) across arms and more importantly high expression of immune gene signatures and low expression of stroma gene signatures were predictive of higher PCR (18). With more sequencing data available, correlative studies should pave for enriching future treatment studies for these patient populations. This would be invaluable for optimization of patient treatment.

\section{ROLE OF NERATINIB IN ADJUVANT TREATMENT}

ExteNET enrolled 2,840 HER2-positive patients who had received standard chemotherapy followed by 1 year of maintenance trastuzumab. These patients were considered high-risk owing to their node positivity or residual disease post neoadjuvant therapy. Patients in the treatment arm received 1 year of neratinib $240 \mathrm{mg} /$ day (Figure 1).

Results of the ExteNET trial suggest an overall benefit of neratinib with a $2.3 \%$ absolute improvement in the 2 -year iDFS (5). The trial underwent amendment to limit enrollment to nodepositive patients (after it already included $24 \%$ node negative in the analysis of the study group). Concordant with the HERA trial where there was an early separation on the iDFS curves in the second year of extended trastuzumab therapy in both hormone positive and negative patients; a similar 2-year separation of curves was observed in the ExteNET trial. However, the benefit was primarily seen in the hormone positive patients (95.4 vs $91.2 \%, P=0.0013)$. Neratinib showed a $2.3 \%$ absolute difference in DFS compared to placebo after 2 years, with stratification for hormone receptor status showing a pronounced HR of 0.51 for ER-positive disease, perhaps resulting from modifying estrogen receptor sensitivity to hormonal agents. Patients with $\geq 4$ positive lymph nodes also achieved an additional benefit reflected in a superior DFS of 97.8 vs $96.5 \%$ at 2 years with HR of 0.65 (0.41-1.01) (5).

These findings support a potential benefit of "pan HER inhibition" in the HR-positive population. Barring the high rate of diarrhea, this TKI would seem a likely next candidate in the HER2 saga, primarily in HR-positive disease. Witton et al. have shown there is a strong interaction, in terms of survival, between HER expression and ER expression and in the HER2-positive tumors, with the curve flattening after 6 years (19). However, whether this translates to a survival improvement is not clear. Neoadjuvant chemotherapy was received in $24 \%$ in the neratinib group and $27 \%$ in the placebo, and no separate analysis was done to only assess patient with neoadjuvant treatment, but they were included in the overall analysis and benefit.

TABLE 2 | Patients benefiting in APHINITY and ExteNET.

\begin{tabular}{|c|c|c|}
\hline Patient characteristic & Neratinib & Pertuzumab \\
\hline DFS & $95.2(\mathrm{HR}+)$ vs 91.2\% (HR-) DFS@ 2 years & 92 vs $90 \% 3$ years iDFS \\
\hline Node positive & HR 0.70 in node + similar to ITT population ${ }^{a}$ & $1.8 \%$ Absolute DFS improvement \\
\hline $\mathrm{HR}+$ & HR 0.51 for HR+ (improved outcome) & HR 0.81 (overall); 0.77 in high-risk node positive \\
\hline $\mathrm{HR}-$ & No benefit & $1.6 \%$ Absolute DFS improvement \\
\hline Mechanism & PAN HER inhibition, MAPK, ERK, AKT downregulation & HER2 inhibition \\
\hline Biomarker candidates & RB1CC1, HER3, FOXO3a, NR3C1, CCND1 & $\begin{array}{l}\text { CD8 TIL, anti-HER2 CD4+ T helper, high HER2 protein, HER2 } \\
\text { and HER3 mRNA levels, PD1 for addition of IO }\end{array}$ \\
\hline Ideal patient & High risk, node positive, $\mathrm{HR}+$ & High risk, node positive, HR- \\
\hline Absolute DFS improvement & 2.3\% Absolute DFS improvement @ 2 years & 0.9\% Absolute iDFS improvement @ 3 years $^{a}$ \\
\hline Cost (USD) & $\$ 120,000 /$ year & $\$ 70,000 /$ year \\
\hline
\end{tabular}

Ideal patient candidates for adjuvant neratinib vs pertuzumab in clinical setting outside of a clinical trial given no comparative studies between the drugs. These are considerations in HER2-positive high-risk patients.

HR, hormone receptor; DFS, disease-free survival; ITT, intention to treat; TIL, tumor-infiltrating lymphocytes; iDFS, invasive disease-free survival.

a2-Year. DFS based on LN status LN neg: 99.4 vs 99.2\%, HR 0.82 (0.32-2.03); LN 1-3: 97.8 vs 96.5\%, HR 0.66 (0.41-1.02); LN $\geq 4$ : DFS 97.8 vs 96.5\%, HR 0.65 (0.41-1.01). 
MAPK pathways may be downregulated by neratinib, and the activity against AKT and ERK could be correlated with efficacy in neratinib $(20,21)$, but these need to be studied in robust correlative studies. Biomarker candidates who have been investigated in vitro for neratinib include upregulation of RB1CC1, HER3, FOXO3a, and NR3C1, as well as downregulation of CCND1 mRNA $(14,22)$.

Taken in context, however, one should not forget the cost issue. The addition of pertuzumab will add approximately US\$70,000/year. The estimated yearly cost of neratinib is US $\$ 120,000$. In addition to the cost of treatment, the clinical toxicity including cardiac toxicity and diarrhea must be considered. Grade 3 diarrhea was seen in up to $40 \%$ patients. The median duration of diarrhea was 5 days and occurred very early mostly during the first month. A protocol amendment mandating antidiarrheal prophylaxis with loperamide in tapering down fashion reduced the grade 3 diarrhea to $17 \%$ in exploratory analysis (5).

For any future studies involving a population with good prognosis, the challenge is to identify the few that benefit from therapy and the majority that do not. As a matter of perspective, other trials in the non-HER2 expressing population have paved the way for fine tuning treatment. Studies on gene expression profiling have established risk categories that identify patients who will have no benefit from chemotherapy. The development and validation of prognostic and predictive tools for the selective use of dual therapy in HER2-positive breast cancer would be a big step in the care of these patients. We need to identify candidates for either drug in practice setting since currently, no comparative data are available to facilitate this decision (Table 2).

\section{REFERENCES}

1. von Minckwitz G, Procter M, de Azambuja E, Zardavas D, Benyunes M, Viale G, et al. Adjuvant pertuzumab and trastuzumab in early HER2-positive breast cancer. N Engl J Med (2017) 377(2):122-31. doi:10.1056/NEJMoa1703643

2. Perez EA, Romond EH, Suman VJ, Jeong JH, Sledge G, Geyer CE Jr, et al. Trastuzumab plus adjuvant chemotherapy for human epidermal growth factor receptor 2-positive breast cancer: planned joint analysis of overall survival from NSABP B-31 and NCCTG N9831. J Clin Oncol (2014) 32: 3744-52. doi:10.1200/JCO.2014.55.5730

3. Slamon D, Eiermann W, Robert N, Pienkowski T, Martin M, Press M, et al. Adjuvant trastuzumab in HER2-positive breast cancer. N Engl J Med (2011) 365(14):1273-83. doi:10.1056/NEJMoa0910383

4. Goldhirsch A, Gelber RD, Piccart-Gebhart MJ, de Azambuja E, Procter M, Suter TM, et al. 2 years versus 1 year of adjuvant trastuzumab for HER2-positive breast cancer (HERA): an open-label, randomised controlled trial. Lancet (2013) 382:1021-8. doi:10.1016/S0140-6736(13) 61094-6

5. Chan A, Delaloge S, Holmes FA, Moy B, Iwata H, Harvey VJ, et al. Neratinib after trastuzumab-based adjuvant therapy in patients with HER2-positive breast cancer (ExteNET): a multicentre, randomised, double-blind, placebocontrolled, phase 3 trial. Lancet Oncol (2016) 17:367-77. doi:10.1016/ S1470-2045(15)00551-3

6. Tolaney SM, Barry WT, Dang CT, Yardley DA, Moy B, Kelly Marcom P, et al. Adjuvant paclitaxel and trastuzumab for node-negative, HER2-positive breast cancer. N Engl J Med (2015) 372:134-41. doi:10.1056/NEJMoa1406281

7. Piccart-Gebhart M, Holmes E, Baselga J, de Azambuja E, Dueck AC, Viale G, et al. Adjuvant lapatinib and trastuzumab for early human epidermal growth factor receptor 2-positive breast cancer: results from the randomized phase III adjuvant lapatinib and/or trastuzumab treatment optimization trial. J Clin Oncol (2016) 34:1034-42. doi:10.1200/JCO.2015.62.1797

\section{CONCLUSION}

The development of HER2 targeted agents resulted in dramatic survival benefits when added to a chemotherapy backbone, a significant stride in the treatment of what was once considered a poor prognosis subgroup. However, it is expected that any additional benefit from newer HER2-targeted agents will provide smaller incremental benefits.

FDA approval of these new HER2-targeted agents does not mean that every patient is a candidate for these treatments. While we find comfort in the growing number of therapeutic options that we once did not have, our enthusiasm in prescribing these newer class of drugs must be balanced with financial pragmatism, anticipated treatment-related toxicities, and a realistic estimation of the degree of benefit for the individual patient. In this regard, the optimal patient selection is still lacking.

Future adjuvant HER2 trials need to use selected rather than unselected populations and should be focused on using less intensive therapy when possible, and on finding biomarkers on secondary analysis. We need to be careful in the presumption that benefits in metastatic disease setting can be translated to the adjuvant setting especially in an unselected population $(23,24)$. For now, we should probably be restricting the use of adjuvant pertuzumab to women with node-positive disease, and considering neratinib in the high risk, hormone receptor-positive population.

\section{AUTHOR CONTRIBUTIONS}

$\mathrm{AB}$ - concept and manuscript writing. EA-M and $\mathrm{ZD}-$ manuscript writing.

8. Schneeweiss A, Chia S, Hegg R, Tausch C, Deb R, Ratnayake J, et al. Evaluating the predictive value of biomarkers for efficacy outcomes in response to pertuzumab- and trastuzumab-based therapy: an exploratory analysis of the TRYPHAENA study. Breast Cancer Res (2014) 16:R73. doi:10.1186/bcr3690

9. Baselga J, Cortés J, Im SA, Clark E, Ross G, Kiermaier A, et al. Biomarker analyses in CLEOPATRA: a phase III, placebo-controlled study of pertuzumab in human epidermal growth factor receptor 2-positive, first-line metastatic breast cancer. J Clin Oncol (2014) 32:3753-61. doi:10.1200/JCO.2013. 54.5384

10. Bianchini G, Kiermaier A, Bianchi GV, Im YH, Pienkowski T, Liu MC, et al. Biomarker analysis of the NeoSphere study: pertuzumab, trastuzumab, and docetaxel versus trastuzumab plus docetaxel, pertuzumab plus trastuzumab, or pertuzumab plus docetaxel for the neoadjuvant treatment of HER2-positive breast cancer. Breast Cancer Res (2017) 19:16. doi:10.1186/s13058-0170806-9

11. Bianchini G, Pusztai L, Pienkowski T, Im YH, Bianchi GV, Tseng LM, et al. Immune modulation of pathologic complete response after neoadjuvant HER2-directed therapies in the NeoSphere trial. Ann Oncol (2015) 26:2429-36. doi:10.1093/annonc/mdv395

12. Stanton SE, Adams S, Disis ML. Variation in the incidence and magnitude of tumor-infiltrating lymphocytes in breast cancer subtypes: a systematic review. JAMA Oncol (2016) 2:1354-60. doi:10.1001/jamaoncol.2016.1061

13. Datta J, Berk E, Xu S, Fitzpatrick E, Rosemblit C, Lowenfeld L, et al. AntiHER2 CD4(+) T-helper type 1 response is a novel immune correlate to pathologic response following neoadjuvant therapy in HER2-positive breast cancer. Breast Cancer Res (2015) 17:71. doi:10.1186/s13058-015-0584-1

14. Feldinger K, Kong A. Profile of neratinib and its potential in the treatment of breast cancer. Breast Cancer (Dove Med Press) (2015) 7:147-62. doi:10.2147/ BCTT.S54414

15. Bose R, Kavuri SM, Searleman AC, Shen W, Shen D, Koboldt DC, et al. Activating HER2 mutations in HER2 gene amplification negative breast 
cancer. Cancer Discov (2013) 3:224-37. doi:10.1158/2159-8290.CD12-0349

16. Jaiswal BS, Kljavin NM, Stawiski EW, Chan E, Parikh C, Durinck S, et al. Oncogenic ERBB3 mutations in human cancers. Cancer Cell (2013) 23:603-17. doi:10.1016/j.ccr.2013.04.012

17. Gianni L, Bianchini G, Valagussa P, Belousov A, Thomas M, Ross G, et al. Abstract S6-7: adaptive immune system and immune checkpoints are associated with response to pertuzumab $(\mathrm{P})$ and trastuzumab $(\mathrm{H})$ in the NeoSphere study. Cancer Res (2012) 72:S6-7. doi:10.1158/0008-5472. SABCS12-S6-7

18. Fumagalli D, Venet D, Ignatiadis M, Azim HA Jr, Maetens M, Rothé F, et al. RNA sequencing to predict response to neoadjuvant anti-HER2 therapy: a secondary analysis of the NeoALTTO randomized clinical trial. JAMA Oncol (2017) 3(2):227-34. doi:10.1001/jamaoncol.2016.3824

19. Witton CJ, Reeves JR, Going JJ, Cooke TG, Bartlett JM. Expression of the HER1-4 family of receptor tyrosine kinases in breast cancer. J Pathol (2003) 200:290-7. doi:10.1002/path.1370

20. Seyhan AA, Varadarajan U, Choe S, Liu Y, McGraw J, Woods M, et al. A genome-wide RNAi screen identifies novel targets of neratinib sensitivity leading to neratinib and paclitaxel combination drug treatments. Mol Biosyst (2011) 7:1974-89. doi:10.1039/c0mb00294a

21. Canonici A, Gijsen M, Mullooly M, Bennett R, Bouguern N, Pedersen K, et al. Neratinib overcomes trastuzumab resistance in HER2 amplified breast cancer. Oncotarget (2013) 4:1592-605. doi:10.18632/oncotarget.1148
22. O’Neill F, Madden SF, Clynes M, Crown J, Doolan P, Aherne ST, et al. A gene expression profile indicative of early stage HER2 targeted therapy response. Mol Cancer (2013) 12:69. doi:10.1186/1476-4598-12-69

23. Kelly K, Chansky K, Gaspar LE, Albain KS, Jett J, Ung YC, et al. Phase III trial of maintenance gefitinib or placebo after concurrent chemoradiotherapy and docetaxel consolidation in inoperable stage III non-small-cell lung cancer: SWOG S0023. J Clin Oncol (2008) 26:2450-6. doi:10.1200/JCO.2007.14.4824

24. Goss GD, O'Callaghan C, Lorimer I, Tsao MS, Masters GA, Jett J, et al. Gefitinib versus placebo in completely resected non-small-cell lung cancer: results of the NCIC CTG BR19 study. J Clin Oncol (2013) 31:3320-6. doi:10.1200/ JCO.2013.51.1816

Conflict of Interest Statement: $\mathrm{AB}$ is on advisory board of Pfizer and Astra Zeneca. The remaining authors declare that the research was conducted in the absence of any commercial or financial relationships that could be construed as a potential conflict of interest.

Copyright (c) 2018 Bulbul, Araujo-Mino and Dayao. This is an open-access article distributed under the terms of the Creative Commons Attribution License (CC BY). The use, distribution or reproduction in other forums is permitted, provided the original author(s) and the copyright owner are credited and that the original publication in this journal is cited, in accordance with accepted academic practice. No use, distribution or reproduction is permitted which does not comply with these terms. 